\title{
Morphokinematic properties of the 21 micron source IRAS $22272+5435$
}

\author{
Jun-ichi Nakashima ${ }^{1}$, Sun Kwok ${ }^{1}$, Bosco H. K. Yung ${ }^{1}$, Yong Zhang ${ }^{1}$, \\ Nico Koning ${ }^{2}$ and Nikolaus Volgenau ${ }^{3}$ \\ ${ }^{1}$ Department of Physics, University of Hong Kong, Pokfulam Rd., Hong Kong, China \\ email: junichi@hku.hk \\ ${ }^{2}$ Department of Physics and Astronomy, University of Calgary, Calgary, Canada T2N 1N4 \\ ${ }^{3}$ Owens Valley Radio Observatory, Big Pine, CA 93513, USA
}

\begin{abstract}
We obtained a high-resolution CO map of IRAS $22272+5435$ in the CO $J=2-1$ line using CARMA. The target exhibits a second biggest angular size of the circumstellar molecular envelope among known $21 \mu \mathrm{m}$ sources. In the preliminary results, we found that the CO properties of IRAS $22272+5435$ is clearly different from those of IRAS $07134+1005$, which is another well-investigated $21 \mu \mathrm{m}$ source. For example, elongations seen in the mid-infrared and CO images are extended in mutually perpendicular directions, although in case of IRAS $07134+1005$ the $\mathrm{CO}$ feature coincides well with the mid-infrared structure.
\end{abstract}

Keywords. stars: AGB and post-AGB, stars: carbon, stars: individual (IRAS 22272+5435), stars: imaging, stars: kinematics, stars: winds, outflows

\section{Introduction}

The target is a proto-typical $21 \mu \mathrm{m}$ source: i.e., a carbon-rich proto-planetary nebula (PPN) with an unidentified mid-IR feature at $21 \mu \mathrm{m}$ (see, e.g., Kwok et al. 1989). The $21 \mu \mathrm{m}$ sources are a fascinating sample to investigate the metamorphosis of intermediatemass evolved stars, because they are lying within a particular narrow evolutionary stage.

A recent radio CO observation of IRAS $07134+1005$, another typical $21 \mu \mathrm{m}$ source, revealed an expanding torus, but did not exhibit a jet (Nakashima et al. 2009), even though a bipolar jet is often detected together with a torus in PPNe. We have suggested that this phenomenon might be a common characteristic of $21 \mu \mathrm{m}$ sources, but of course we need to investigate more $21 \mu \mathrm{m}$ sources in radio $\mathrm{CO}$ lines to compare the morphokinematic properties. However, the angular size of the circumstellar envelopes of $21 \mu \mathrm{m}$ sources is intrinsically too small to be resolved by conventional radio interferometers. So far, only IRAS $07134+1005$ has been resolved in previous radio CO mapping.

\section{Results and Discussion}

In this project, we have obtained a high-resolution CO map of IRAS 22272+5435 using the Combined Array for Research in Millimeter-wave Astronomy (CARMA). The target exhibits a second biggest size of the circumstellar envelope among known $21 \mu \mathrm{m}$ sources. In the preliminary results, we found that the CO properties of IRAS $22272+5435$ are clearly different from those of IRAS $07134+1005$. For example, elongations seen the midinfrared and CO images are extended in mutually perpendicular directions, although in case of IRAS $07134+1005$ the CO feature coincides well with the mid-infrared structure. Notable results are summarized as follows: 

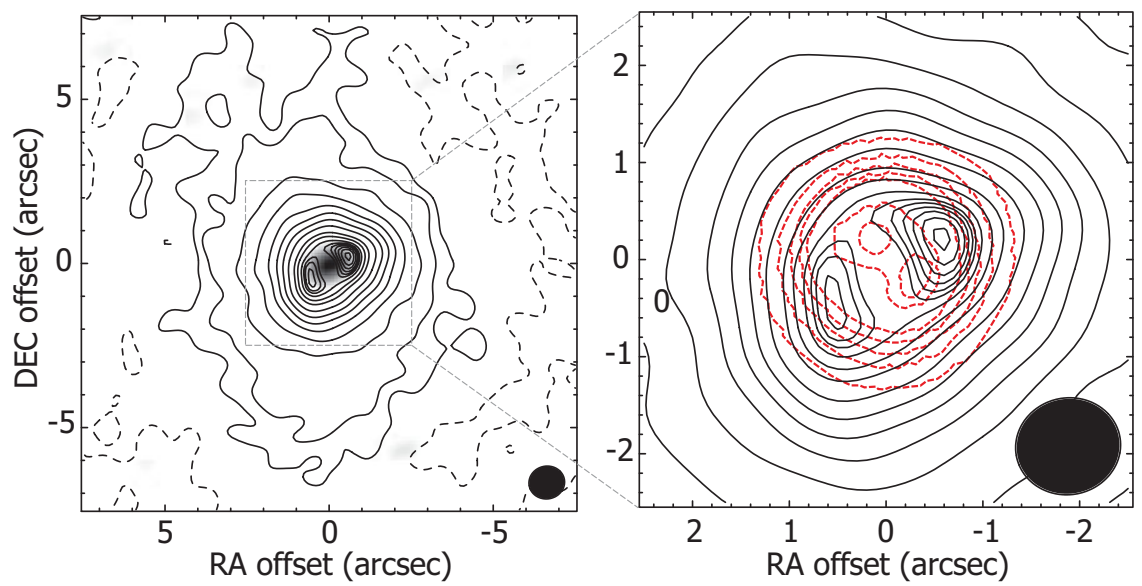

Figure 1. Left: total flux intensity map in the ${ }^{12} \mathrm{CO} J=2-1$ line superimposed on the $1 \mathrm{~mm}$ radio continuum image (gray scale). The contour levels are $5,10,20,30,40,50,60,70,80,85,89$, $91,92,93,94,95$ and $96 \sigma$, and the $1 \sigma$ level corresponds to $1.01 \times 10^{-2} \mathrm{Jy} \mathrm{beam}^{-1}$. The dashed contour correspond to $-3 \sigma$. The FWHM beam size is located in the bottom right corner. Right: total flux intensity map in the ${ }^{12} \mathrm{CO} J=2-1$ line superimposed on the mid-infrared $12.5 \mu \mathrm{m}$ image (red dashed contour) taken from Ueta et at. (2001). The contour levels of the CO image are the same as the left panel.

(1) The CO structure is elongated in the north-west to south-east direction, and we see separated two intensity peaks along with this direction in the velocity integrated intensity map (see, Figure 1). This characteristic is reminiscent of the CO $J=3-2$ map of IRAS $07134+1005$, which can be explained with an expanding torus (and expanding sphere; Nakashima et al. 2009).

(2) However, the resolved CO structure of IRAS $22272+5435$ is out of synchronization with the $12.5 \mu \mathrm{m}$ structure (see right panel of Fig. 1). In fact, the elongation of the $\mathrm{CO}$ structure is perpendicular to that of the $12.5 \mu \mathrm{m}$ structure. (But, we see a week correlation with the optical $I$-band image, though we do not present the optical image here.)

(3) In case of IRAS $22272+5435$, the CO $J=2-1$ line and $12.5 \mu \mathrm{m}$ emission might trace different parts of a torus, because those may trace different temperatures. Otherwise, we might see the onset of a molecular jet, which may complicate the $\mathrm{CO}$ and mid-IR structure. Careful morpho-kinematic modeling of the CO data may help to consider this problem (Nakashima et al., in preparation).

\section{Acknowledgements}

This work is supported by a grant awarded to Jun-ichi Nakashima from the Research Grants Council of Hong Kong (project code: HKU 704209P; HKU 704710P; HKU 704411P) and the Small Project Funding of the University of Hong Kong (project code: 201007176004).

\section{References}

Kwok, S., Volk, K., \& Hrivnak, B. J. 1989, ApJ, 345, L51

Nakashima, J., Koning, N., Kwok, S., \& Zhang, Y. 2009, ApJ, 692, 402

Ueta, T., Meixner, M., Hinz, P. M., et al. 2001, ApJ, 557, 831 